IoAnNis K. Argyros (Lawton, OK)

\title{
NEW UNIFYING CONVERGENCE CRITERIA FOR NEWTON-LIKE METHODS
}

Abstract. We present a local and a semilocal analysis for Newton-like methods in a Banach space. Our hypotheses on the operators involved are very general. It turns out that by choosing special cases for the "majorizing" functions we obtain all previous results in the literature, but not vice versa. Since our results give a deeper insight into the structure of the functions involved, we can obtain semilocal convergence under weaker conditions and in the case of local convergence a larger convergence radius.

1. Introduction. In this study we are concerned with the problem of approximating a locally unique solution $x^{*}$ of the equation

$$
F(x)=0,
$$

where $F$ is a Fréchet-differentiable operator defined on a closed convex subset $D$ of a Banach space $X$ with values in a Banach space $Y$.

Most problems in applied sciences (like systems of equations, differential equations, integral equations, integro-differentiable equations and others) can be brought in the form (1) for a suitable choice of the corresponding spaces.

Newton-like methods

$$
x_{n+1}=x_{n}-A\left(x_{n}\right)^{-1} F\left(x_{n}\right) \quad(n \geq 0)
$$

have been used extensively under various hypotheses to generate a sequence converging to $x^{*}[3]-[7],[9],[10],[17],[18]$.

We present local and semilocal convergence theorems under very general conditions on the operators involved. This way we can handle a wider range

2000 Mathematics Subject Classification: 65B05, 47H17, 49M15, 65G99, 65J15, 65N30, $65 \mathrm{~N} 35$.

Key words and phrases: Banach space, Newton-like method, Fréchet derivative, majorizing sequence, non-differentiable operator, convergence radius. 
of problems than was possible before. Moreover by choosing special cases for the "majorizing" functions, we obtain all previous results in the literature but not vice versa. Furthermore since our results give a deeper insight into the structure of the functions involved, we can in particular obtain semilocal convergence under weaker conditions and in the case of local convergence a larger convergence radius.

2. Semilocal convergence analysis. It is convenient for us to define the non-negative number $a$ by

$$
\left\|A\left(x_{0}\right)^{-1} F\left(x_{0}\right)\right\| \leq a
$$

for some $x_{0} \in D$ such that $A\left(x_{0}\right)^{-1} \in L(Y, X)$.

We suppose

$$
\left\|A\left(x_{0}\right)^{-1}\left[F^{\prime}(y)-A(x)\right]\right\| \leq w_{1}(\|y-x\|)+v_{1}\left(\left\|x-x_{0}\right\|\right)+v_{0}
$$

for all $x, y \in \bar{U}\left(x_{0}, R\right)=\left\{x \in X:\left\|x-x_{0}\right\| \leq R\right\}$ and $R \geq 0$ such that

$$
\bar{U}\left(x_{0}, R\right) \subseteq D,
$$

where $v_{1}, w_{1}:[0, \infty) \rightarrow[0, \infty)$ are increasing, $v_{0} \in[0,1)$,

$$
\begin{aligned}
& \left\|A\left(x_{0}\right)^{-1}\left[A(x)-A\left(x_{0}\right)\right]\right\| \leq w_{0}\left(\left\|x-x_{0}\right\|\right) \\
& \quad \text { for all } x \in \bar{U}\left(x_{0}, r\right) \quad(0 \leq r \leq R),
\end{aligned}
$$

where again $w_{0}:[0, \infty) \rightarrow[0, \infty)$ is increasing and

$$
\lim _{r \rightarrow 0} w_{1}(r)=\lim _{r \rightarrow 0} v_{1}(r)=\lim _{r \rightarrow 0} \bar{w}_{0}(r)=0,
$$

with $w_{0}(r)=\bar{w}_{0}(r)+\bar{v}_{0}, r \in[0, R]$, for some $\bar{v}_{0} \in[0,1)$. Define functions on $[0, R]$ by

$$
\begin{gathered}
w(r)=\sup \left\{w_{1}\left(t_{1}\right)+v_{1}\left(t_{2}\right)+v_{0}+w_{0}\left(t_{2}\right):\right. \\
\left.t_{1}+t_{2}=r, t_{1} \geq 0, t_{2} \geq 0\right\}, \\
\varphi(r)=\int_{0}^{r} w(t) d t-r+a
\end{gathered}
$$

and a sequence

$$
r_{0}=0, \quad r_{n+1}=r_{n}+\frac{\varphi\left(r_{n}\right)}{1-w_{0}\left(r_{n}\right)} \quad(n \geq 0) .
$$

With the notation introduced above we can show the following semilocal convergence theorem for Newton-like methods:

TheOREm 1. Suppose that a function $\varphi$ has a unique zero $r^{*} \in[0, R]$ and $\varphi(R) \leq 0$. Then:

(i) The scalar sequence $\left\{r_{n}\right\}(n \geq 0)$ converges monotonically to $r^{*}$. 
(ii) A Newton-like method $\left\{x_{n}\right\}(n \geq 0)$ is well defined, remains in $\bar{U}\left(x_{0}, r^{*}\right)$ for all $n \geq 0$ and converges to a solution $x^{*}$ of the equation $F(x)$ $=0$. Moreover the following error bounds hold for all $n \geq 0$ :

$$
\left\|x_{n+1}-x_{n}\right\| \leq r_{n+1}-r_{n}
$$

and

$$
\left\|x^{*}-x_{n}\right\| \leq r^{*}-r_{n} .
$$

(iii) If $r^{*}=R$ then the solution $x^{*}$ is unique in $\bar{U}\left(x_{0}, r^{*}\right)$ provided that

$$
w_{1}\left(r^{*}\right)<1 \text {. }
$$

If $r^{*}<R$ then the solution $x^{*}$ is unique in $U\left(x_{0}, R_{0}\right)$ provided that

$$
R_{0} \leq R \quad \text { and } \quad \int_{0}^{1} w_{1}\left[t R_{0}+(1-t) r^{*}\right] d t<1 .
$$

Proof. (i) This follows exactly as in the proof of Lemma 1 in [12, p. 214] (see also [10], [19]).

In particular note that since $r^{*}$ is the only zero of $\varphi$ on $[0, R]$, this function is strictly positive on $\left[0, r^{*}\right)$. The same is true for the function $r \rightarrow 0<-\varphi^{\prime}(r) \leq 1-w_{0}(r)$ (by (8)). Moreover the map

$$
r \mapsto r+\frac{\varphi(r)}{1-w_{0}(r)}
$$

is increasing on $\left[0, r^{*}\right.$ ) (as can easily be seen by showing the non-negativity of the derivative). The rest of the proof is standard.

(ii) We show (11) and (12) by induction on $n \geq 0$. For $n=0$ inequality (11) holds as equality. Indeed,

$$
\left\|x_{1}-x_{0}\right\|=\left\|A\left(x_{0}\right)^{-1} F\left(x_{0}\right)\right\| \leq a \leq r_{1}=r_{1}-r_{0} .
$$

Suppose (11) holds for all $n<k$. Then we have

$$
\left\|x_{k}-x_{0}\right\| \leq \sum_{j=1}^{k}\left\|x_{j}-x_{j-1}\right\| \leq \sum_{j=1}^{k}\left(r_{j}-r_{j-1}\right)=r_{k} \leq r^{*} .
$$

That is, $x_{k} \in \bar{U}\left(x_{0}, r_{k}\right) \subseteq \bar{U}\left(x_{0}, r^{*}\right)$.

Using (6) and (9) we obtain

$$
\left\|A\left(x_{0}\right)^{-1}\left[A\left(x_{k}\right)-A\left(x_{0}\right)\right]\right\| \leq w_{0}\left(\left\|x_{k}-x_{0}\right\|\right) \leq w_{0}\left(r_{k}\right)<1
$$

(see part (i)). It follows by (15) and the Banach lemma on invertible operators [14] that $A\left(x_{k}\right)^{-1}$ exists and

$$
\left\|A\left(x_{k}\right)^{-1} A\left(x_{0}\right)\right\| \leq\left[1-w_{0}\left(\left\|x_{k}-x_{0}\right\|\right)\right]^{-1} \leq\left[1-w_{0}\left(r_{k}\right)\right]^{-1} .
$$

Moreover, by (2), (4)-(10) and (16) we obtain in turn 
(17)

$$
\begin{aligned}
& \left\|x_{k+1}-x_{k}\right\| \\
= & \left\|A\left(x_{k}\right)^{-1} F\left(x_{k}\right)\right\| \leq\left\|A\left(x_{k}\right)^{-1} A\left(x_{0}\right)\right\| \cdot\left\|A\left(x_{0}\right)^{-1} F\left(x_{k}\right)\right\| \\
\leq & \left\|A\left(x_{k}\right)^{-1} A\left(x_{0}\right)\right\| \\
& \times\left\|A\left(x_{0}\right)^{-1}\left[F\left(x_{k}\right)-F\left(x_{k-1}\right)-A\left(x_{k-1}\right)\left(x_{k}-x_{k-1}\right)\right]\right\| \\
\leq & \left\|A\left(x_{k}\right)^{-1} A\left(x_{0}\right)\right\| \\
& \times \int_{0}^{1}\left\|F^{\prime}\left[x_{k}+t\left(x_{k}-x_{k-1}\right)\right]-A\left(x_{k-1}\right)\right\| \cdot\left\|x_{k}-x_{k-1}\right\| d t \\
\leq & \frac{1}{1-w_{0}\left(r_{k}\right)} \\
& \times \int_{0}^{1}\left\{w_{1}\left[r_{k-1}+t\left(r_{k}-r_{k-1}\right)-r_{k-1}\right]+v_{1}\left(r_{k-1}\right)+v_{0}\right\}\left(r_{k}-r_{k-1}\right) d t \\
\leq & \frac{1}{1-w_{0}\left(r_{k}\right)} \int_{0}^{1}\left\{w\left(r_{k-1}+t\left(r_{k}-r_{k-1}\right)\right)-w_{0}\left(r_{k-1}\right)\right\}\left(r_{k}-r_{k-1}\right) d t \\
\leq & \frac{\varphi\left(r_{k}\right)-\varphi\left(r_{k-1}\right)-\left(w_{0}\left(r_{k-1}\right)-1\right)\left(r_{k}-r_{k-1}\right)}{1-w_{0}\left(r_{k}\right)}=r_{k+1}-r_{k},
\end{aligned}
$$

which shows (11) for $n=k$. Furthermore, for $m>n$ we get

$$
\left\|x_{m}-x_{n}\right\| \leq r_{m}-r_{n} .
$$

It follows from $(18)$ that $\left\{x_{n}\right\}(n \geq 0)$ is a Cauchy sequence in the Banach space $X$ and hence it converges to some $x^{*} \in \bar{U}\left(x_{0}, r^{*}\right)$ (since $\bar{U}\left(x_{0}, r^{*}\right)$ is a closed set). By letting $n \rightarrow \infty$ in (2) we obtain $F\left(x^{*}\right)=0$ and by letting $m \rightarrow \infty$ in (18) we obtain (12).

Finally to show uniqueness, let $y^{*} \in U\left(x_{0}, R_{0}\right)$ be a solution of equation (1). We have, by (4),

$$
\begin{aligned}
\int_{0}^{1} \| A\left(x_{0}\right)^{-1}\left[F^{\prime}(x+t(y-x))-\right. & \left.A\left(x_{0}\right)\right] \| d t \\
& \leq \int_{0}^{1} w_{1}\left[\left\|x+t(y-x)-x_{0}\right\|\right] d t \\
& \leq \int_{0}^{1} w_{1}\left[t\left\|y-x_{0}\right\|+(1-t)\left\|x-x_{0}\right\|\right] d t \\
& \leq \int_{0}^{1} w_{1}\left[t R_{0}+(1-t) r^{*}\right] d t<1 .
\end{aligned}
$$


Hence, the inverse of the linear operator

$$
L=\int_{0}^{1} F^{\prime}\left(x^{*}+t\left(y^{*}-x^{*}\right)\right) d t
$$

exists. But we can write

$$
0=F\left(y^{*}\right)-F\left(x^{*}\right)=L\left(y^{*}-x^{*}\right),
$$

which shows that under (13) or (14),

$$
x^{*}=y^{*} \text {. }
$$

REMARK 1. (a) Let $A(x)=F^{\prime}(x)(x \in D)$ and set

$$
\bar{v}_{0}=v_{0}=0, \quad v_{1}(r)=0 \quad(0 \leq r \leq R) .
$$

Then Theorem 1 reduces to Theorem 2 in [12, p. 215]. Moreover, if we choose $w_{1}(r)=w_{0}(r), r \in[0, R]$, we obtain the main theorem in [1]. Note however that uniqueness results are not given in either [1] or [12]. Furthermore, choose

$$
w_{1}(r)=\ell r, \quad r \in[0, R], \ell>0 .
$$

It follows by (8) that

$$
w(r)=\ell r, \quad r \in[0, R],
$$

and (9) becomes

$$
\varphi_{1}(r)=\frac{1}{2} \ell r^{2}-r+a .
$$

In this case the function $\varphi$ has a zero if and only if

$$
2 \ell a \leq 1 \text {. }
$$

We set

$$
R=\frac{1}{\ell} \quad \text { and } \quad r^{*}=\frac{1-\sqrt{1-2 \ell a}}{\ell} .
$$

Condition (19) is the Newton-Kantorovich hypothesis for the convergence of Newton's method given in [14].

(b) Assume there exist increasing functions $w_{2}, w_{3}:[0, \infty) \rightarrow[0, \infty)$ such that

$$
\begin{aligned}
\left\|A\left(x_{0}\right)^{-1}\left[F^{\prime}(y)-F^{\prime}(x)\right]\right\| & \leq w_{2}(\|y-x\|), \\
\left\|A\left(x_{0}\right)^{-1}\left[F^{\prime}(x)-A(x)\right]\right\| & \leq w_{3}\left(\left\|x-x_{0}\right\|\right)+v_{0}
\end{aligned}
$$

for all $x, y \in \bar{U}\left(x_{0}, r\right)$, and

$$
\lim _{r \rightarrow 0} w_{2}(r)=\lim _{r \rightarrow 0} w_{3}(r)=0 .
$$

Define functions on $[0, R]$ by

$$
\begin{aligned}
& w_{4}(r)=\sup \left\{w_{2}\left(t_{1}\right)+w_{0}\left(t_{2}\right): t_{1}+t_{2}=r, t_{1} \geq 0, t_{2} \geq 0\right\} \\
& \varphi_{2}(r)=\int_{0}^{r} w_{4}(t) d t+r w_{3}(r)+v_{0} r-r+a
\end{aligned}
$$


and an iteration

$$
s_{0}=0, \quad s_{n+1}=s_{n}+\frac{\varphi_{2}\left(s_{n}\right)}{1-w_{0}\left(s_{n}\right)} \quad(n \geq 0) .
$$

Based on the identity

$$
\begin{aligned}
F\left(x_{k}\right)= & \int_{0}^{1}\left[F^{\prime}\left(x_{k-1}+t\left(x_{k}-x_{k-1}\right)\right)-F^{\prime}\left(x_{k-1}\right)\right]\left(x_{k}-x_{k-1}\right) d t \\
& +\left[F^{\prime}\left(x_{k-1}\right)-A\left(x_{k-1}\right)\right]\left(x_{k}-x_{k-1}\right)
\end{aligned}
$$

instead of (17), the conclusions of Theorem 1 hold if we replace $\varphi,\left\{r_{n}\right\}$ by $\varphi_{2},\left\{s_{n}\right\}(n \geq 0)$ respectively. Moreover, if we set, for all $x, y \in \bar{U}\left(x_{0}, r\right)$, $\ell_{i} \geq 0, i=1, \ldots, 5$,

$$
\begin{aligned}
w_{2}(\|y-x\|) & =\ell_{1}\|y-x\|, & & \\
w_{3}\left(\left\|x-x_{0}\right\|\right) & =\ell_{2}\left\|x-x_{0}\right\|, & & \ell_{3}=v_{0}, \\
w_{0}\left(\left\|x-x_{0}\right\|\right) & =\ell_{4}\left\|x-x_{0}\right\|+\ell_{5}, & \ell_{5} & =\bar{v}_{0},
\end{aligned}
$$

we obtain the theorem due to Yamamoto [17]. For $A(x)=F^{\prime}(x)(x \in D)$ and $w_{3}=0$ we again obtain Theorem 2 in [12].

(c) Consider the iteration

$$
x_{n+1}=x_{n}-A\left(x_{n}\right)^{-1}\left(F\left(x_{n}\right)+G\left(x_{n}\right)\right) \quad(n \geq 0)
$$

for approximating a solution of the equation

$$
F(x)+G(x)=0,
$$

where $F$ is as in Theorem 1 and $G: D \rightarrow Y$ is a continuous operator.

Assume there exists a continuous, non-decreasing function $w_{5}:[0, \infty) \rightarrow$ $[0, \infty)$ such that

$$
\left\|A\left(x_{0}\right)^{-1}[G(x)-G(y)]\right\| \leq w_{5}(r)\|x-y\|
$$

for all $x, y \in \bar{U}\left(x_{0}, r\right)$, and set

$$
w_{6}(r)=\int_{0}^{r} w_{5}(t) d t
$$

Define a function $\varphi_{3}$ by

$$
\varphi_{3}(r)=w_{6}(r)+\int_{0}^{r} w(t) d t-r+a
$$

and an iteration $\left\{b_{n}\right\}(n \geq 0)$ by

$$
b_{0}=0, \quad b_{n+1}=b_{n}+\frac{\varphi_{3}\left(b_{n}\right)}{1-w_{0}\left(b_{n}\right)} \quad(n \geq 0) \text {. }
$$


Then the conclusions of Theorem 1 concerning (26) and (27) hold with $\varphi$, $\left\{r_{n}\right\}$ replaced by $\varphi_{3},\left\{b_{n}\right\}(n \geq 0)$ respectively. If we let

$$
\begin{aligned}
w_{0}\left(\left\|x-x_{0}\right\|\right) & =w_{7}\left(\left\|x-x_{0}\right\|\right)+c_{0}, \\
w_{1}(r+t) & =w_{8}(r+t)-w_{7}(r)+c_{1}, \quad t \geq 0, r \in[0, R],
\end{aligned}
$$

for some $c_{0}, c_{1} \geq 0$ and continuous non-decreasing functions $w_{7}, w_{8}(r+t)-$ $w_{7}(r)(t \geq 0)$, then we obtain the main theorem in [10].

(d) Theorem 1 can be further generalized (similarly for cases (a)-(c)) if instead of (4) we assume that

$$
\begin{aligned}
& \left\|A\left(x_{0}\right)^{-1}\left[F^{\prime}(y)-A(x)\right]\right\| \\
& \quad \leq v_{2}\left(\|x-y\|,\left\|x-x_{0}\right\|,\left\|y-x_{0}\right\|\right)+v_{3}\left(\left\|x-x_{0}\right\|\right)+v_{4}\left(\left\|y-x_{0}\right\|\right)+v_{5}
\end{aligned}
$$

for all $x, y \in \bar{U}\left(x_{0}, r\right) \subseteq \bar{U}\left(x_{0}, R\right)$, where $v_{2}:[0, \infty)^{3} \rightarrow[0, \infty), v_{3}, v_{4}:[0, \infty)$ $\rightarrow[0, \infty)$ are increasing, $v_{5} \in[0,1)$ and

$$
\lim _{r \rightarrow 0} v_{2}(r, r, r)=\lim _{r \rightarrow 0} v_{3}(r)=\lim _{r \rightarrow 0} v_{4}(r)=0 .
$$

Define functions on $[0, R]$ by

$$
\begin{aligned}
v(r)= & \sup \left\{v_{2}\left(t_{1}, t_{2}, t_{1}\right)+v_{3}\left(t_{2}\right)+v_{4}\left(t_{1}\right)+v_{5}+w_{0}\left(t_{2}\right):\right. \\
& \left.t_{1}+t_{2}=r, t_{1} \geq 0, t_{2} \geq 0\right\}, \\
\varphi_{3}(r)= & \int_{0}^{r} v(r)-r+a
\end{aligned}
$$

and a sequence

$$
p_{0}=0, \quad p_{n+1}=p_{n}+\frac{\varphi_{3}\left(p_{n}\right)}{1-w_{0}\left(p_{n}\right)} \quad(n \geq 0) .
$$

Then the conclusions of Theorem 1 hold with $\varphi,\left\{s_{n}\right\}$ replaced by $\varphi_{3},\left\{p_{n}\right\}$ $(n \geq 0)$. If we let

$$
v_{2}(r, r, r)+v_{3}(r)+v_{4}(r)+v_{5}=w_{0}(r), \quad r \in[0, R],
$$

we essentially obtain the results in [5].

(e) Consider a Newton-like method (2) in the form

$$
x_{n+1}=x_{n}-A\left(x_{n}\right)^{\#} F\left(x_{n}\right) \quad(n \geq 0)
$$

for solving the equation

$$
A^{\#} F(x)=0 \text {, }
$$

where a $A^{\#}$ is a bounded outer inverse of $A\left(x_{0}\right)$, and $A\left(x_{n}\right)^{\#}$ denotes an outer inverse of $A\left(x_{n}\right)$, i.e., $A\left(x_{n}\right)^{\#} A\left(x_{n}\right) A\left(x_{n}\right)^{\#}=A\left(x_{n}\right)^{\#}(n \geq 0)$. The conclusions of Theorem 1 hold for (29) and (30) if we simply replace $A\left(x_{0}\right)$ by $A^{\#}$ in the hypotheses of the theorem, and set

$$
A\left(x_{n}\right)^{\#}=\left[I+A^{\#}\left(A\left(x_{n}\right)-A\left(x_{0}\right)\right)\right]^{-1} A^{\#}\left(x_{0}\right),
$$


with uniqueness holding in $\bar{U}\left(x_{0}, r^{*}\right) \cap\left\{R\left(A^{\#}\left(x_{0}\right)\right)+x_{0}\right\}$, where

$$
R\left(A^{\#}\left(x_{0}\right)\right)+x_{0}=\left\{x+x_{0}: x \in R\left(A^{\#}\left(x_{0}\right)\right)\right\} .
$$

In the special case when the functions $w_{2}, w_{3}, w_{0}$ are given by $(23),(24),(25)$ respectively, we obtain Theorem 3.1 in [9, p. 241] concerning the convergence of Newton-like methods using outer inverses.

(f) With the exception of the uniqueness part (iii) in Theorem 1 the rest of the conclusions hold if we replace condition (4) by the Mysovskilu-type condition

$$
\begin{aligned}
\int_{0}^{1}\left\|A\left(x_{0}\right)^{-1}\left[F^{\prime}(x+t(y-x))-A(x)\right](y-x)\right\| d t & \\
\leq & {\left[\bar{w}_{1}(\|y-x\|)+\bar{v}_{1}\left(\left\|x-x_{0}\right\|\right)+\overline{\bar{v}}_{0}\right]\|y-x\| }
\end{aligned}
$$

for all $x, y \in \bar{U}\left(x_{0}, R\right)$, where $\bar{w}_{1}, \bar{v}_{1}, \overline{\bar{v}}_{0}$ are like $w_{1}, v_{1}, v_{0}$ respectively. Since $\|L(x)\| \leq\|L\| \cdot\|x\|$ for any linear operator $L$, it is expected that $\bar{w}_{1} \leq w_{1}$, which leads to smaller error bounds on the distances $\left\|x_{n+1}-x_{n}\right\|(n \geq 0)$.

3. Local convergence analysis. We complete this study by showing the following local convergence theorem for Newton-like methods.

ThEOREM 2. Suppose there exist: a simple zero $x^{*} \in D$ of the equation $F(x)=0$ with $A\left(x^{*}\right)^{-1} \in L(Y, X)$; increasing functions $\alpha, \alpha_{1}, \beta:[0, \infty) \rightarrow$ $[0, \infty)$ with

$$
\lim _{r \rightarrow 0} \alpha(r)=\lim _{r \rightarrow 0} \alpha_{1}(r)=\lim _{r \rightarrow 0} \beta(r)=0 ;
$$

and non-negative parameters $\alpha_{0}, \beta_{0}$ with $\alpha_{0}+\beta_{0} \in[0,1)$ such that

$$
\begin{aligned}
\left\|A\left(x^{*}\right)^{-1}\left[F^{\prime}(y)-A(x)\right]\right\| & \leq \alpha(\|y-x\|)+\alpha_{1}\left(\left\|x-x^{*}\right\|\right)+\alpha_{0}, \\
\left\|A\left(x^{*}\right)^{-1}\left[A(x)-A\left(x^{*}\right)\right]\right\| & \leq \beta\left(\left\|x-x^{*}\right\|\right)+\beta_{0}
\end{aligned}
$$

for all $x, y \in D$. Suppose also that the equation

$$
\int_{0}^{1} \alpha(t r) d t+\beta(r)+\alpha_{1}(r)+\alpha_{0}+\beta_{0}-1=0
$$

has a minimum non-negative zero $R_{1}$ such that

$$
\bar{U}\left(x^{*}, R_{1}\right) \subseteq D .
$$

Then the Newton-like method $\left\{x_{n}\right\}(n \geq 0)$ generated by (2) is well defined, remains in $U\left(x^{*}, r\right)\left(r<R_{1}\right)$ for all $n \geq 0$ and converges to $x^{*}$ provided that $x_{0} \in U\left(x^{*}, r\right)$. Moreover the following error bounds hold for all $n \geq 1$ :

$$
\left\|x_{n}-x^{*}\right\| \leq \gamma_{n}\left\|x_{n-1}-x^{*}\right\|,
$$


where

$$
\gamma_{n}=\frac{\int_{0}^{1} \alpha\left(t\left\|x^{*}-x_{n-1}\right\|\right) d t+\alpha_{1}\left(\left\|x^{*}-x_{n-1}\right\|\right)+\alpha_{0}}{1-\left[\beta\left(\left\|x^{*}-x_{n-1}\right\|\right)+\beta_{0}\right]} .
$$

Proof. Clearly, $x_{0} \in U\left(x_{0}, r\right)$. Assume $x_{k-1} \in U\left(x_{0}, r\right)$ for all $k<n$. Using (32) and (33) we obtain

$$
\left\|A\left(x^{*}\right)\left(A\left(x_{k}\right)-A\left(x^{*}\right)\right)\right\| \leq \beta\left(\left\|x_{k}-x^{*}\right\|\right)+\beta_{0} \leq \beta\left(R_{1}\right)+\beta_{0}<1 .
$$

It follows from (37) and the Banach lemma on invertible operators that $A\left(x_{k}\right)^{-1}$ exists and

$$
\left\|A\left(x_{k}\right)^{-1} A\left(x^{*}\right)\right\| \leq \frac{1}{1-\left[\beta\left(\left\|x_{k}-x^{*}\right\|\right)+\beta_{0}\right]} \leq \frac{1}{1-\left[\beta\left(R_{1}\right)+\beta_{0}\right]} .
$$

Using (2) we get in turn

$$
\begin{aligned}
x_{k}-x^{*}= & x_{k-1}-x^{*}-A\left(x_{k-1}\right)^{-1} F\left(x_{k-1}\right) \\
= & A\left(x_{k-1}\right)^{-1}\left[F\left(x^{*}\right)-F\left(x_{k-1}\right)-A\left(x_{k-1}\right)\left(x^{*}-x_{k-1}\right)\right] \\
= & {\left[A\left(x_{k-1}\right)^{-1} A\left(x^{*}\right)\right] A\left(x^{*}\right)^{-1} } \\
& \quad \cdot \int_{0}^{1}\left[F^{\prime}\left(x_{k-1}+t\left(x^{*}-x_{k-1}\right)\right)-A\left(x_{k-1}\right)\right]\left(x^{*}-x_{k-1}\right) d t .
\end{aligned}
$$

Moreover, by (31), (38) and (39) we obtain

$\left\|x_{k}-x^{*}\right\|$

$$
\begin{aligned}
& \leq\left\|A\left(x_{k-1}\right)^{-1} A\left(x^{*}\right)\right\| \\
& \quad \times \int_{0}^{1} \| A\left(x^{*}\right)^{-1}\left[F^{\prime}\left(x_{k-1}+t\left(x^{*}-x_{k-1}\right)\right)-A\left(x_{k-1}\right)\|\cdot\| x^{*}-x_{k-1} \| d t\right. \\
& \leq \gamma_{k}\left\|x^{*}-x_{k-1}\right\|,
\end{aligned}
$$

which shows (35) for all $n \geq 1$. Furthermore, by the choice of $R_{1}, r$ and by (33) there exists

$$
\gamma=\frac{\int_{0}^{1} \alpha\left(t r_{0}\right) d t+\alpha_{1}\left(r_{0}\right)+\alpha_{0}}{1-\left[\beta\left(r_{0}\right)+\beta_{0}\right]} \in[0,1)
$$

such that

$$
\gamma_{k} \leq \gamma \quad(k \geq 1)
$$

That is,

$$
\left\|x_{k}-x^{*}\right\| \leq \gamma\left\|x_{k-1}-x^{*}\right\| \leq \gamma^{k}\left\|x_{0}-x^{*}\right\| \leq \gamma^{k} r \quad(k \geq 1) .
$$

It follows from $(40)$ that $x_{k} \in U\left(x^{*}, r\right)$ and $\lim _{n \rightarrow \infty} x_{n}=x^{*}$ (since $\gamma \in$ $[0,1))$. 
Remark 2. Let $A(x)=F^{\prime}(x)(x \in D)$. Choose:

$$
\alpha(\|x-y\|)=\delta_{1}\|x-y\|, \quad \alpha_{1}(r)=0, \quad r \in\left[0, R_{1}\right), \quad \alpha_{0}=0,
$$

and

$$
\beta\left(\left\|x-x^{*}\right\|\right)=\delta_{2}\left\|x-x^{*}\right\|, \quad \beta_{0}=0 .
$$

Consequently, equation (33) becomes

$$
\frac{1}{2} \delta_{1} r+\delta_{2} r-1=0
$$

which gives

$$
R_{1}=\frac{2}{\delta_{1}+2 \delta_{2}} .
$$

It was shown in [16] that the convergence radius for Newton's method under the Lipschitz condition:

$$
\left\|F^{\prime}\left(x^{*}\right)^{-1}\left[F^{\prime}(y)-F^{\prime}(x)\right]\right\| \leq \delta_{1}\|y-x\|
$$

for all $x, y \in D$, is given by

$$
\bar{R}_{1}=\frac{2}{3 \delta_{1}}
$$

However, since

$$
\delta_{2} \leq \delta_{1} \quad \text { (in general) }
$$

we obtain

$$
\bar{R}_{1} \leq R_{1} .
$$

Let $X=Y=\mathbb{R}, D=U(0,1)$ and define a function $F$ on $D$ by

$$
F(x)=e^{x}-1 .
$$

Then it can easily be seen that $\delta_{1}=e$ and $\delta_{2}=e-1$. Consequently, we obtain

$$
\bar{R}_{1}=.245252961<R_{1}=.254028662 .
$$

That is, we obtain a wider range of initial guesses $x_{0}$ than before. This observation is very important in the construction of very efficient mesh refinement strategies and in projection methods [3], [8], [15], [18].

Note that several other local convergence theorems can be generated along the lines of Theorem 1 and Remark 1.

\section{References}

[1] J. Appell, E. De Pascale, J. V. Lysenko and P. P. Zabrejko, New results on NewtonKantorovich approximations with applications to nonlinear integral equations, $\mathrm{Nu}-$ mer. Funct. Anal. Optim. 18 (1997), 1-17.

[2] J. Appell, E. De Pascale, and P. P. Zabrejko, On the application of the NewtonKantorovich method to nonlinear integral equations of Uryson-type, ibid. 12 (1991), 271-283. 
[3] I. K. Argyros, A convergence theorem for Newton-like methods under generalized Chen-Yamamoto-type assumptions, Appl. Math. Comput. 61 (1994), 25-37.

[4] - On the discretization of Newton-like methods, Int. J. Comput. Math. 52 (1994), $161-170$.

[5] -, On a new Newton-Mysovskir-type theorem with applications to inexact Newtonlike methods and their discretizations, IMA J. Numer. Anal. 18 (1998), 37-56.

[6] -, A Newton-Kantorovich theorem for equations involving m-Fréchet differentiable operators and applications in radiative transfer, J. Comput. Appl. Math. 131 (2001), $149-159$.

[7] I. K. Argyros and F. Szidarovszky, The Theory and Applications of Iteration Methods, CRC Press, Boca Raton, FL, 1993.

[8] P. N. Brown, A local convergence theory for combined inexact-Newton/finite-difference projection methods, SIAM J. Numer. Anal. 24 (1987), 407-434.

[9] X. Chen and M. Z. Nashed, Convergence of Newton-like methods for singular operator equations using outer inverses, Numer. Math. 66 (1993), 235-257.

[10] X. Chen and T. Yamamoto, Convergence domains of certain iterative methods for solving nonlinear equations, Numer. Funct. Anal. Optim. 10 (1989), 37-48.

[11] P. Deuflhard and G. Heindl, Affine invariant convergence theorems for Newton's method and extensions to related methods, SIAM J. Numer. Anal. 16 (1979), 1-10.

[12] E. De Pascale and P. P. Zabrejko, New convergence criteria for the Newton-Kantorovich method and some applications to nonlinear integral equations, Rend. Sem. Mat. Univ. Padova 100 (1998), 211-230.

[13] J. M. Gutiérrez, A new semilocal convergence theorem for Newton's method, J. Comput. Appl. Math. 79 (1997), 131-145.

[14] L. V. Kantorovich and G. P. Akilov, Functional Analysis, Pergamon Press, Oxford, 1982.

[15] F. A. Potra, On Q-order and R-order of convergence, J. Optim. Theory Appl. 63 (1989), 415-431.

[16] W. C. Rheinboldt, An adaptive continuation process for solving systems of nonlinear equations, in: Banach Center Publ. 3, PWN, 1977, 129-142.

[17] T. Yamamoto, A convergence theorem for Newton-like methods in Banach spaces, Numer. Math. 51 (1987), 545-557.

[18] T. J. Ypma, Local convergence of inexact Newton methods, SIAM J. Numer. Anal. 21 (1984), 583-590.

[19] P. P. Zabrejko and D. F. Nguen, The majorant method in the theory of NewtonKantorovich approximations and the Pták error estimates, Numer. Funct. Anal. Optim. 9 (1987), 671-684.

Department of Mathematics

Cameron University

Lawton, OK 73505, U.S.A.

E-mail: ioannisa@cameron.edu

Received on 8.3.2002;

revised version on 13.6.2002 